\title{
Year in Review 2018: Lung Cancer
}

\author{
Revisão Ano 2018: Câncer de Pulmão \\ Vladmir Cláudio Cordeiro de Lima ${ }^{1,2}$, Clarissa Baldotto ${ }^{1,3}$, Ricardo Mingarini Terra ${ }^{4}$, Livia Fagundes ${ }^{5}$, \\ Ana Gelatti ${ }^{1,6}$, William Nassib William Jr ${ }^{1,5}$
}

\begin{abstract}
Over the past decade, significant progress has been achieved for clinical management of lung cancers, including screening strategies, use of immunotherapy-based approaches to treat locally advanced and metastatic disease, and development of novel targeted agents for molecularly defined subgroups. In this review, we summarize the studies published/presented in 2018 that form the basis for new standard-of-care management options for lung cancers. These practice-changing studies exemplify the importance of multidisciplinary, evidence-based care to improve outcomes for lung cancer patients.
\end{abstract}

Keywords: Lung Neoplasms; Immunotherapy; Molecular Targeted Therapy

\section{RESUMO}

Durante a última década, foi alcançado um progresso significativo no tratamento clínico de câncer de pulmão, incluindo estratégias de rastreamento, uso de abordagens baseadas em imunoterapia para tratar a doença localmente avançada e a metastática, e o desenvolvimento de novos agentes direcionados para subgrupos definidos molecularmente. Nesta revisão, resumimos os estudos publicados/apresentados em 2018 que formam a base para novas opções de tratamento padrão para o câncer de pulmão. Esses estudos que mudam a prática exemplificam a importância de cuidados multidisciplinares baseados em evidências para melhorar os resultados para pacientes com câncer de pulmão.

Descritores: Neoplasias Pulmonares; Imunoterapia; Terapia Molecular Direcionada

1. Grupo Brasileiro de Oncologia Torácica, Grupo Brasileiro de Oncologia Torácica - Porto Alegre - RS - Brazil.

2. AC Camargo Cancer Center, Departamento de Oncologia - São Paulo - SP - Brazil.

3. Oncologia D’Or, Departamento de Oncologia - Rio de Janeiro - RJ - Brazil.

4. Faculdade de Medicina da Universidade de São Paulo, Instituto do Câncer do Estado de São Paulo - São Paulo - SP - Brazil.

5. Beneficência Portuguesa de São Paulo, Centro Oncológico - São Paulo - SP - Brazil.

6. Hospital do Câncer Mãe de Deus, Departamento de Oncologia - Porto Alegre - RS - Brazil.

Financial support: none to declare.

Conflicts of interest: Dr. William has received honoraria/speaker's fee and/or participated in advisory boards from Roche/Genentech, BMS, Eli Lilly, Merck, AstraZeneca, and Pfizer.

Corresponding author: William N. William Jr. Centro Oncológico BP, a Beneficência Portuguesa de São Paulo. Rua Martiniano de Carvalho, 965, 2º Andar, São Paulo, SP, Brazil. Zip-code: 01323-001. E-mail: williamwilliamjr@gmail.com

Received on: November 28, 2018 | Accepted on: November 29, 2018

DOI: $10.5935 / 1806-6054.20180007$ 


\section{INTRODUCTION}

Despite extensive clinical investigations, lung cancer remains the leading cause of cancer-related deaths worldwide, ${ }^{(1)}$ illustrating the need for novel approaches to reduce lung cancer incidence and mortality. Lately, significant progress has been achieved for clinical management of lung cancers, including screening strategies, use of immunotherapy-based approaches to treat locally advanced and metastatic disease, and development of novel targeted agents for molecularly defined subgroups. Much of the data that support these strategies became available just within the past year. In this review, we summarize the studies published/presented in 2018 that form the basis for new standard-of- care management options for lung cancers. These practice-changing studies exemplify the importance of multidisciplinary, evidence-based care to improve outcomes for lung cancer patients.

\section{Computerized tomography (CT) scans for lung cancer screening}

The role of lung cancer screening with low dose chest CT scans was evaluated by the NELSON trial.(2) NELSON was a randomized clinical trial from The Netherlands and Leuven that compared, in a high-risk population, the lung cancer mortality reduction associated with CT-screening versus no screening. An important feature of the NELSON trial was the fact that investigators utilized a nodule volume management strategy in contrast with nodule diameter, used in previous studies like NLST. ${ }^{(3)}$

The study population was selected based on the answers of a mail questionnaire sent to 606,409 persons aged 50-74, of which 15,822 eligible participants gave informed consent and were eventually randomized (1:1). Eligibility criteria included smokers with a smoking history of $>10$ cigarettes/day for $>30$ years or $>15$ cigarettes/day for $>25$ years, and, in former smokers, smoking cessation in $\leq 10$ years. CT-screening was offered to study arm participants at baseline and 1, 3 and 5.5 years after randomization. Participants' records were linked to national registries and an expert panel blinded to study arm reviewed medical files for deceased lung cancer patients. CT screening compliance was 94\% on average. In $9.1 \%$ of the participants, additional CT scans within 2 months were performed to estimate nodule volume doubling time, leading to an overall referral rate of $2.1 \%$ for suspicious nodules. Detection rates across the rounds varied between 0.8-1.0\%, and $69 \%$ of screen-detected lung cancers were detected at stage IA or B. There were 214 lung cancer deaths in the control arm and 157 in the screen arm. The lung cancer mortality rate ratio at Year 10 was 0.74 (0.60-0.91). The lung cancer mortality rate ratio observed at Year 10 was 0.61 in the small subset of female patients. NELSON confirmed previous NLST findings by demonstrating the capacity of CT screening to reduce lung cancer mortality in a high-risk population of smokers. Moreover, it also suggested that the nodule volume management strategy was associated with less false positives as well as the fact that lung cancer screening might be more effective in females.

\section{Overall survival with durvalumab after chemora- diotherapy in stage III non-small cell lung cancer (NSCLC)}

Approximately one third of NSCLC patients present with stage III disease. The standard treatment for the past several years has been concurrent platinum- based chemoradiation therapy, resulting in a median progression-free survival of 8 months and 5 -year overall survival of 15\%. PACIFIC was the first phase III study to evaluate consolidation immunotherapy for patients with stage III, unresectable NSCLC. In 2017, the initial results of the PACIFIC trial demonstrated that consolidation therapy with the anti-PD-L1 antibody durvalumab tripled the median progression-free survival (5.6 months versus 16.8 months) in stage III, unresectable NSCLCs who had not progressed after concurrent chemoradiation therapy. ${ }^{(4)}$ Response rates were also higher in the durvalumab group (28\% versus $16 \%$ ). Toxicity was similar between the two groups, but the main causes of treatment interruption were pneumonia and pneumonitis, underscoring the importance of radiation therapy delivery quality. Based on these results, the FDA and ANVISA approved durvalumab consolidation therapy, now considered a new standard of care. In November 2018, updates on the PACIFIC trial were published, with a median follow-up time of 25.2 months. ${ }^{(5)}$ There were improvements in the 24-month overall survival in favor of the durvalumab arm (34.7 months versus 28.7 months), as well as a lower incidence of distant metastases, including a $50 \%$ reduction in the development of new brain lesions. The benefits in progression-free, and overall survival occurred in all subgroups, irrespective of histology or PD-L1 expression levels ( $<$ or $\geq 25 \%$ ). Controversies on the use of durvalumab still remain, such as the optimal timing for initiation of therapy. In PACIFIC, durvalumab was started 1-42 days after completion of chemoradiation; a subgroup analysis demonstrated improved progression-free, and overall survival, and time to distant metastases when durvalumab was initiated within 14 days of chemoradiation therapy. The PACIFIC trial results provide evidence for pivotal interactions between radiation therapy and immunotherapy, and support an important change in the standard treatment of stage III NSCLC, after a decade's time without significant advances in this field.

\section{Pembrolizumab plus chemotherapy in metastatic, non-squamous, NSCLC}

The combination of chemotherapy and immunotherapy for treatment-naïve non- squamous, NSCLC was investigated in the KEYNOTE-189 trial. ${ }^{(6)}$ In this study, 616 patients with EGFR wild-type, ALK-negative 
cancers were stratified according to PD-L1 expression levels, platinum compound, and smoking history, and were randomized to receive platinum (carboplatin or cisplatin), pemetrexed, and placebo for up to 4 cycles, followed by maintenance pemetrexed and placebo, versus platinum, pemetrexed, pembrolizumab for up to 4 cycles, followed by maintenance pemetrexed and pembrolizumab. Crossover from the placebo to the pembrolizumab arm was allowed at the time of disease progression. The primary endpoints of the study (progression-free and overall survival) were met. The 12-month overall survival was $69.2 \%$ in the pembrolizumab arm versus $49.4 \%$ in the placebo arm (hazard ratio [HR] 0.49, $95 \% \mathrm{Cl}$ 0.38-0.64, $\mathrm{P}<0.001)$. The median progression-free survival was 8.8 months versus 4.9 months for the pembrolizumab and placebo groups, respectively ( $\mathrm{HR} 0.52,95 \% \mathrm{Cl} 0.43-0.64, \mathrm{P}<0.001)$. Response rates were also higher in the pembrolizumab arm (47.6\% versus $18.9 \%, \mathrm{P}<0.0001)$. Improvements in overall survival, progression-free survival and response rates occurred in all three subgroups defined by PD-L1 status (i.e., tumor proportion score [TPS] $<1 \%, 1-49 \%$, and $\geq 50 \%)$. Nonetheless, greatest benefits were observed among the patients whose tumors had higher PD-L1 levels - HR (95\% Cl) for overall survival in PD-L1 TPS $<1 \%, 1-49 \%$, and $\geq 50 \%$ were 0.59 (0.38-0.92), 0.55 (0.34-0.90), and $0.42(0.26$ $0.68)$, respectively. Grade 3-5 adverse events were observed in $67.2 \%$ and $65.8 \%$ of patients assigned to the pembrolizumab and placebo groups, respectively. Acute kidney injury (all grades) happened in 5.2\% of pembrolizumab-treated patients versus $0.5 \%$ of placebo-treated patients, and should be carefully screened for in routine clinical practice. Taken together, these data confirmed the hypothesis that the combination of chemotherapy and anti-PD1 antibodies improves outcomes in metastatic NSCLCS, and defined platinum, pemetrexed, and pembrolizumab as a new standard of care for treatment naïve, EGFR wild-type, and ALK-negative non-squamous NSCLCS, irrespective of PD-L1 expression levels.

\section{Pembrolizumab plus chemotherapy for squamous NSCLC}

Squamous cell carcinomas correspond to $20 \%$ of lung cancers and is associated with a shorter survival. Treatment for this disease has not greatly improved in the last 15 years. The KEYNOTE-407 trial randomized 559 stage IV squamous NSCLC patients with measurable disease and ECOG performance status 0 or 1, to receive carboplatin plus paclitaxel/ nab-paclitaxel every 21 days for 4 cycles associated with pembrolizumab or placebo every 21 days for 35 cycles or until disease progression or unacceptable toxicity. Patients with symptomatic central nervous system metastasis, pneumonitis requiring corticosteroids, systemic immunosuppressive treatment, or active auto-immune disease were excluded. Patients in the placebo arm were allowed to cross over to receive pembrolizumab after confirmed disease progression. The trial had two co-primary endpoints: overall survival and progression-free survival.(7) The median follow-up time was 7.8 months. PD-L1 TPS $\geq 1 \%$ was observed in $63 \%$ of patients. Median overall survival was 15.8 months in the pembrolizumab arm and 11.3 months in the placebo group (HR for death $0.64 ; 95 \% \mathrm{Cl}, 0.49-0.85 ; \mathrm{p}<0.001$ ). Median progression-free survival was 6.4 months in the pembrolizumab arm and 4.8 months in the placebo arm (HR for progression or death 0.56; 95\% Cl, 0.45-0.70; $\mathrm{p}<0.001)$. The combination with pembrolizumab was also associated with improved overall response rate (ORR) (57.9\% versus 38.4\%) and duration of response (7.7 months versus 4.8 months). The benefit of the addition of pembrolizumab to chemotherapy in overall survival, progression-free survival and response rates was observed for all the prespecified PD-L1 TPS subgroups, and increased with every increment of PD- L1 expression. Adverse events of grade $\geq 3$ occurred in $69.8 \%$ in the pembrolizumab group and $68.2 \%$ in the placebo group, leading to death in $8.3 \%$ and $6.8 \%$ of patients, respectively. Immune mediated adverse events or infusion reactions of grade $\geq 3$ were observed in $10.8 \%$ and $3.2 \%$ of the pembrolizumab and placebo-treated patients, respectively. In view of the dismal outcomes with current treatment for metastatic squamous NSCLCs, the results of KEYNOTE-407 confirmed the rationale to combine pembrolizumab with carboplatin and paclitaxel or nab-paclitaxel and established a new paradigm in the treatment of this disease.

\section{Brigatinib for frontline treatment of ALK positive NSCLC}

Brigatinib is a next-generation ALK inhibitor that targets a broad range of ALK mutations. ALTA- $1 \mathrm{~L}$ was an open-label, multicenter, randomized, phase 3 trial comparing the efficacy and safety of brigatinib with crizotinib in patients with ALK-positive NSCLC who had not received previous treatment with an ALK inhibitor. ${ }^{(8)}$ Patients with asymptomatic, untreated CNS metastases were not excluded. In the crizotinib group, crossover to brigatinib was permitted after progression. The primary endpoint was progression-free survival. In this study, 275 patients were enrolled. Brigatinib showed superior efficacy against extra- and intra-cranial disease. With a median follow-up of 11.0 months in the brigatinib group and 9.3 months in the crizotinib group, brigatinib was associated with a $51 \%$ lower risk of disease progression or death than crizotinib (HR 0.49; $\mathrm{P}<0.001$ ). The 12-month progression-free survival was $67 \%$ in the brigatinib group (median not reached) and $43 \%$ in the crizotinib group. The confirmed objective response rate was $71 \%(95 \% \mathrm{Cl}, 62$ to 78$)$ with brigatinib and $60 \%(95 \% \mathrm{Cl}, 51$ to 68$)$ with crizotinib; the confirmed intracranial response rate among patients with measurable lesions was $78 \%$ (95\% Cl, 52 to 94$)$ and $29 \%(95 \% \mathrm{Cl}, 11$ to 52$)$, respectively. ${ }^{(9)}$ No new safety concerns were noted. Despite shorter follow-up, these initial ALTA- $1 \mathrm{~L}$ results appear similar to results from the phase 3 ALEX trial, which compared alectinib versus crizotinib. A key strength of 
this trial is that a blinded independent review committee assessed progression-free survival, and ALK was assessed locally, increasing the applicability of these data. A limitation is that overall survival data will be confounded by crossover of patients and subsequent use of other tyrosine kinase inhibitors after trial. In conclusion, brigatinib is a new first line option to treat ALK-positive NSCLC.

\section{First-line atezolizumab plus chemotherapy in extensive small-cell lung cancer (SCLC)}

The combination of chemotherapy and immunotherapy for treatment-naïve advanced SCLC was investigated in the IMpower-133 trial. ${ }^{(10)}$ In this doubleblind, placebo-controlled, phase 3 study, a total of 403 ECOG PS 0-1 patients with extensive SCLC were randomized to receive carboplatin and etoposide with either atezolizumab (an anti-PD-L1 inhibitor) or placebo for four 21-day cycles (induction phase), followed by a maintenance phase during which they received either atezolizumab or placebo. Crossover was not allowed. Patients with treated asymptomatic brain metastases were eligible. During the maintenance phase, prophylactic cranial irradiation was permitted, but thoracic radiation therapy was not.
PD-L1 testing was not performed during screening. Median follow-up was 13.9 months. The primary endpoints of the study, overall survival and progression-free survival, were met. The median overall survival was 12.3 months in the atezolizumab group and 10.3 months in the placebo group (HR 0.70; 95\% Cl: 0.54 to $0.91 ; p=0.007)$. The median progression-free survival was 5.2 months and 4.3 months, respectively (HR 0.77; 95\% Cl: 0.62 to $0.96 ; p=0.02$ ). Objective response rate (60.2\% versus $64.4 \%)$ and duration of response were similar in the two groups. The benefit was consistent across key evaluated subgroups (sex, age, ECOG PS, brain and liver metastases, blood- based tumor mutational burden). The safety profile was consistent with previously reported data for each individual agent, with no new findings. Immune-related adverse events occurred in $39.9 \%$ in the atezolizumab group and in $24.5 \%$ in the placebo group, with rash and hypothyroidism being the most common. Despite some criticism related to the magnitude of the benefit, this was the first trial, in more than two decades, to show a survival improvement with the addition of a new drug to advanced SCLC first-line treatment. These data are likely to establish the combination of platinum, etoposide and atezolizumab as a new standard of care for this population.

\section{REFERENCES}

1. Bray F, Ferlay J, Soerjomataram I, Siegel RL, Torre LA, Jemal A. Global cancer statistics 2018: GLOBOCAN estimates of incidence and mortality worldwide for 36 cancers in 185 countries. CA Cancer J Clin. 2018;68(6):394-424.

2. De Koning $\mathrm{H}$, Van Der Aalst C, Ten Haaf $\mathrm{K}$, Oudkerk M. PL02.05 Effects of Volume CT Lung Cancer Screening: Mortality Results of the NELSON Randomised-Controlled Population Based Trial. J Thorac Oncol. 2018;13(10):S185.

3. National Lung Screening Trial Research Team, Aberle DR, Adams AM, Berg CD, Black WC, Clapp JD, Fagerstrom RM, et al. Reduced lung-cancer mortality with low-dose computed tomographic screening. N Engl J Med. 2011;365(5):395-409.

4. Antonia SJ, Villegas A, Daniel D, Vicente D, Murakami S, Hui R, et al.; PACIFIC Investigators. Durvalumab after Chemoradiotherapy in Stage III Non-Small-Cell Lung Cancer. N Engl J Med. 2017;377(20):1919-29.

5. Antonia SJ, Villegas A, Daniel D, Vicente D, Murakami S, Hui R, et al.; PACIFIC Investigators. Overall Survival with Durvalumab after Chemoradiotherapy in Stage III NSCLC. N Engl J Med. 2018 Sep 25. DOI: 10.1056/NEJMoa1809697. [Epub ahead of print].
6. Gandhi L, Rodriguez-Abreu D, Gadgeel S, Esteban E, Felip E, De Angelis F, et al.; KEYNOTE-189 Investigators. Pembrolizumab plus Chemotherapy in Metastatic Non-Small-Cell Lung Cancer. N Engl J Med. 2018;378(22):2078-92.

7. Paz-Ares L, Luft A, Vicente $D$, Tafreshi A, Gümüş M, Mazières J, et al.; KEYNOTE-407 Investigators. Pembrolizumab plus Chemotherapy for Squamous Non-Small-Cell Lung Cancer. N Engl J Med. 2018;379(21):2040-51.

8. Camidge DR, Kim HR, Ahn MJ, Yang JC, Han JY, Lee JS, et al. Brigatinib versus Crizotinib in ALKPositive Non-Small-Cell Lung Cancer. N Engl J Med. 2018;379(21):2027-39.

9. Popat S, Kim HR, Ahn MJ, Yang JC, Han JY, Hochmair MJ, et al. Intracranial efficacy of brigatinib (BRG) vs crizotinib (CRZ) in the phase III ALTA-1L trial. Ann Oncol. 2018;29(Suppl 8):mdy424.070.

10. Horn L, Mansfield AS, Szczęsna A, Havel L, Krzakowski M, Hochmair MJ, et al.; IMpower133 Study Group. First-Line Atezolizumab plus Chemotherapy in Extensive-Stage Small-Cell Lung Cancer. N Engl J Med. 2018 Sep 25. DOI: 10.1056/ NEJMoa1809064. [Epub ahead of print] 